\title{
Type of cardioplegic solution as a factor influencing the clinical outcome of open-heart congenital procedures
}

\author{
Michał Sobieraj ${ }^{1}$, Marta Kilanowska ${ }^{1}$, Piotr Ładziński ${ }^{1}$, Irina Garbuzowa ${ }^{2}$, Michał Wojtalik ${ }^{1}$, Jerzy Moczko ${ }^{3}$, \\ Wojciech Mrówczyński ${ }^{1}$
}

\author{
${ }^{1}$ Department of Pediatric Cardiac Surgery, Chair of Cardio-Thoracic Surgery, Poznan University of Medical Sciences, Poznan, \\ Poland \\ 2Pediatric Cardiac Surgery Students' Interest Group, Poznan University of Medical Sciences, Poznan, Poland \\ ${ }^{3}$ Department of Computer Science and Statistics, Poznan University of Medical Sciences, Poznan, Poland
}

Kardiochirurgia i Torakochirurgia Polska 2018; 15 (2): 86-94

\begin{abstract}
Introduction: Cardioplegia is one of the most important modalities of myocardial protection during heart surgery. Aim: To assess the impact of blood cardioplegia on postoperative variables, in comparison with two types of crystalloid cardioplegic solutions during pediatric heart surgery.

Material and methods: One thousand one hundred and twenty-nine patients underwent surgical correction of congenital heart disease with cardioplegia administration between 2006 and 2012. Nonlinear regression models of postoperative low cardiac output syndrome (LCOS) incidence, catecholamine index and total complication count were developed using a genetic algorithm. The Akaike information criterion was applied for selection of the best model. The following explanatory variables were evaluated: cardioplegia type (ST - Saint Thomas, $n=440$; FR - Fresenius, $n=432$; BL - Calafiore, $n=257$ ), congenital heart diseases (CHD) type, age, sex, genetic disorder presence, body surface area (BSA), cardiopulmonary bypass (CBP) time, aortic cross-clamp time, operation urgency, redo surgery, surgeon.

Results: Low cardiac output syndrome presence and higher than average catecholamine indexes were negatively influenced by use of crystalloid cardioplegia (ST or FR), presence of specific CHDs, redo surgery and prolonged CBP time. Increased complication count was related to: crystalloid cardioplegia, presence of specific CHDs, redo surgery, urgency of operation, operation time and CBP time. Higher BSA had a protective effect against higher catecholamine index and increased complication count. Older age was protective against LCOS.

Conclusions: Cardioplegic solutions type influences postoperative variables in children after heart surgery by the negative impact of crystalloid cardioplegia. Blood cardioplegia presents potential advantages for patients - its application may reduce the incidence of low cardiac output syndrome and related complications.
\end{abstract}

Key words: blood cardioplegia, crystalloid cardioplegia, complications.

\section{Streszczenie}

Wstęp: Kardioplegia jest jedną z najważniejszych metod ochrony mięśnia sercowego podczas operacji kardiochirurgicznych.

Cel: Analiza wpływu zastosowania podczas operacji kardiochirurgicznch u dzieci kardiopleginy krwistej na wybrane okołooperacyjne parametry kliniczne w porównaniu z dwoma rodzajami kardioplegin krystalicznych.

Materiat i metody: W latach 2006-2012 u 1129 pacjentów wykonano korekcję chirurgiczną wrodzonej wady serca z użyciem kardioplegii. Przy zastosowaniu algorytmu genetycznego utworzono nieliniowe modele regresji zachowania zmiennych [obecność zespołu niskiego rzutu serca (LCOS), indeks katecholaminowy, całkowita liczba powikłań]. Do selekcji najlepszego modelu regresji użyto kryterium informacyjnego Akaikego. Oceniano następujące zmienne objaśniające: rodzaj kardioplegii (ST - Saint Thomas, $n=440$; FR - Fresenius, $n=432$; BL-Calafiore, $n=257)$, rodzaj wrodzonej wady serca (CHD), wiek, płeć, obecność wad genetycznych, powierzchnia ciała (BSA), czas krążenia pozaustrojowego (CBP), czas zakleszczenia aorty, pilność operacji, ponowna operacja, chirurg.

Wyniki: Zespół niskiego rzutu serca oraz indeks katecholaminowy wyższy od mediany w populacji były związane z zastosowaniem kardioplegii krystalicznej (ST lub FR), obecnością specyficznej wrodzonej wady serca, ponowną operacją oraz przedłużonym czasem krążenia pozaustrojowego. Większa liczba powikłań wiązała się z zastosowaniem kardioplegii krystalicznej, obecnością specyficznej wrodzonej wady serca, ponowną operacją, pilnością operacji, czasem operacji oraz czasem krążenia pozaustrojowego. Większa powierzchnia ciała chronita przed wystąpieniem wyższego indeksu katecholaminowego oraz większą liczbą powikłań. Starszy wiek pacjenta zabezpieczał przed wystąpieniem zespołu niskiego rzutu serca.

Wnioski: Rodzaj kardioplegii zastosowanej podczas operacji kardiochirurgicznych u dzieci wptywa na wybrane zmienne pooperacyjne. Zaznaczony jest negatywny wpływ kardioplegii krystalicznej. Zastosowanie kardioplegii krwistej może przynieść korzyści w postaci zmniejszenia częstości występowania zespołu niskiego rzutu serca oraz związanych z nim powiklań.

Słowa kluczowe: kardioplegia krwista, kardioplegia krystaliczna, powikłania. 


\section{Introduction}

Controlled intraoperative cardiac arrest is indispensable in the majority of pediatric cardiac surgery procedures. One of the most important myocardial protection techniques is cardioplegia - indirect or direct administration of cardioplegic solution to the coronary arteries. Both crystalloid cardioplegia and blood cardioplegia are widely used in pediatric cardiac surgery [1-3]. The choice of cardioplegic solution depends on the surgeon's preference or on the local institutional policy [4].

The advantages of crystalloid cardioplegia are the following: relatively low price, simplicity of use and myocardial protection up to $2 \mathrm{~h}$ after a single dose $[1,5]$. Potential advantages of blood cardioplegia include: oxygen transfer by the red blood cells to the myocardium, free radical scavenging properties and physiologic osmotic pressure that protects against myocardial edema $[1,6]$.

The experience of many units shows clinical advantages of blood cardioplegia administration. However, the previous studies did not give an unequivocal answer as to which cardioplegic solution is the most beneficial (for all congenital cardiac malformations and all age groups of patients).

\section{Aim}

The aim of this study was to assess the impact of blood cardioplegia on postoperative clinical variables, in comparison with two types of cold crystalloid cardioplegic solutions, during open heart surgery in children.

\section{Material and methods \\ Patients}

One thousand one hundred and twenty-nine consecutive patients underwent surgical correction of different congenital heart diseases (CHD) with the use of cardiopulmonary bypass and cardioplegic solution administration in the Department of Pediatric Cardiac Surgery, Poznan University of Medical Sciences, between 2006 and 2012.

Medical records (surgical reports, case histories, local medical database and local data from National Cardiac Surgery Registry - "KROK") were reviewed.

The most common cardiac malformations operated on during this period are presented in Table I. Demographic data according to the type of used cardioplegic solution are presented in Table II.

This observational study has been approved by the local Ethics Committee (resolution: 75/12 from 05.01.2012).

\section{Cardioprotection}

Patients were divided into three groups according to cardioplegia type. Cold crystalloid St. Thomas cardioplegia (ST) was used from 03.01.2006 to 01.03.2008 and was administered to 440 patients. Cold crystalloid Fresenius cardioplegia (FR) was used from 02.03.2008 to 12.11.2011 and was received by 432 patients. Cold blood Calafiore cardioplegia $(\mathrm{BL})$ use was initiated in our department on 25.08.2010 and was administered during 257 operations.
The composition of each cardioplegic solution in given in Table III. Throughout the whole study period all types of cardioplegic solutions were administered in the indirect (via the aortic root) or direct antegrade way.

Administration of cold crystalloid cardioplegia (at $4^{\circ} \mathrm{C}$ ) to the coronary arteries was performed by an external, pneumatic system operated by the anesthesiologist. The solution was delivered under the pressure of no more than $150 \mathrm{~mm} \mathrm{Hg}$ every $30 \mathrm{~min}$. The first dose was $10 \mathrm{ml} / \mathrm{kg}$ patient's body weight, and the next doses were $5 \mathrm{ml} / \mathrm{kg}$ patient's body weight.

The administration of cold blood cardioplegia $\left(4^{\circ} \mathrm{C}\right)$ to the coronary arteries was performed by an additional pump of the heart-lung machine with a heat exchanger operated by the perfusionist. Cardioplegia infusion was under the pressure of no more than $150 \mathrm{~mm} \mathrm{Hg}$ every $30 \mathrm{~min}$ for 3 to $3.5 \mathrm{~min}$.

\section{Statistical methods}

Multivariable regression models were created to assess the co-influence of specific types of cardioplegia on selected clinical variables (with the binominal function or Poisson function). The dependent outcome variables were the following parameters:

- 30-day mortality - "Death_30d",

- the low cardiac output syndrome (defined as acidosis and oliguria occurrence accompanied by the necessity of inotropic drug support and vasodilatation drug administration) - "LCOS",

- delayed sternal closure - "DSC",

- mechanical ventilation time longer than the median value of the entire cohort (18 h) - "INTUB_time",

- intensive care unit stay longer than the median value for all patients (4 days) - "ICU_LOS",

Table I. List of operated cardiac malformations during 2006-2012

\begin{tabular}{lc} 
Cardiac disease & Number of patients (\% of occurrence) \\
ASD II & $260(23)$ \\
\hline VSD & $230(20)$ \\
\hline AVSD & $148(13.1)$ \\
\hline ToF & $111(9.8)$ \\
\hline TGA & $81(7.2)$ \\
\hline AS/AI & $81(7.2)$ \\
\hline UVH & $58(5.1)$ \\
\hline AVVI & $46(4.1)$ \\
\hline TAPVR & $20(1.8)$ \\
\hline MIX & $94(8.7)$ \\
\hline
\end{tabular}

ASD II - atrial septal defect, VSD - ventricular septal defect, AVSD atrioventricular septal defect, ToF - tetralogy of Fallot, TGA - all spectrum of transposition of great arteries, AS/AI - aortic valve malformations (complex and simple), UVH - all spectrum of single ventricle disease, including hypoplastic left heart syndrome, AVVI - isolated atrioventricular valve malformations, TAPVR - all spectrum of total anomalous pulmonary venous return, MIX - other, less frequent cardiac malformations. 
Table II. Demographic data according to type of used cardioplegic solution

\begin{tabular}{|c|c|c|c|}
\hline Parameter & FR & ST & $\mathrm{BL}$ \\
\hline \multicolumn{4}{|l|}{ Demographic variables: } \\
\hline$N$ & 432 & 440 & 257 \\
\hline Gender (female/male) & $204 / 228$ & $207 / 233$ & $103 / 154$ \\
\hline Age [days] & $295(77-741)$ & $313(82-967)$ & 366 (116-999) \\
\hline Body weight [kg] & $7.2(4.1-11.5)$ & $7.5(4.1-13)$ & $8.6(4.9-13.5)$ \\
\hline Body surface area $\left[\mathrm{m}^{2}\right]$ & $0.37(0.25-0.52)$ & $0.38(0.24-0.57)$ & $0.41(0.28-0.59)$ \\
\hline Down's syndrome & 53 & 49 & 31 \\
\hline \multicolumn{4}{|l|}{ Intraoperative variables: } \\
\hline $\mathrm{CBP}[\min ]$ & $83(52-123)$ & $89(60-126)$ & $81(50-118)$ \\
\hline Ao-x-clamp [min] & $42(24-66)$ & $45(27-62)$ & $43(24-61)$ \\
\hline \multicolumn{4}{|l|}{ Postoperative variables: } \\
\hline ICU time [days] & $4(3-9)$ & $4(3-8)$ & $4(3-8)$ \\
\hline Mechanical ventilation [h] & $18(7-73)$ & $22(8-75)$ & $12(7-39)$ \\
\hline 30-day mortality (\%) & 14.9 & 14.9 & 8.9 \\
\hline
\end{tabular}

FR - Fresenius crystalloid cardioplegic solution, ST - St. Thomas crystalloid cardioplegic solution, BL - Calafiore cold blood cardioplegic solution,

$\mathrm{CBP}$ - cardiopulmonary bypass time, Ao-x-clamp - aortic cross-clamp time, ICU time - intensive care unit stay time. Data presented as median and ranges.

Table III. Chemical composition of crystalloid component of cardioplegic solutions

\begin{tabular}{lccc} 
Component $\left[\mathrm{mM} / \mathrm{dm}^{3}\right]$ & $\mathrm{ST}$ & $\mathrm{FR}$ & $\mathrm{BL}$ \\
$\mathrm{K}^{+}$ & 16.37 & 5 & 670.74 \\
\hline $\mathrm{Cl}^{-}$ & 2.28 & 60.92 & 475.5 \\
\hline $\mathrm{Na}^{+}$ & 93.94 & 19.61 & 154 \\
\hline $\mathrm{Ca}^{+}$ & 16.66 & 0.97 & - \\
\hline $\mathrm{SO}_{4}{ }^{2-}$ & 1.21 & - & - \\
\hline $\mathrm{H}_{2} \mathrm{PO}_{4}^{-}$ & 1.28 & - & - \\
\hline Mannitol & 200.03 & 200.03 & - \\
\hline Magnesium DL-hydrogen aspartate & - & 1.6 & 57.71 \\
\hline $6-$ Methylprednisolone & - & 0.67 & - \\
\hline Sodium bicarbonate & 25 & 25 & - \\
\hline Gentamycin & - & 0.042 & - \\
\hline $\mathrm{pH}$ & $4-6$ & $5-7$ & $7.35-7.45$ \\
\hline
\end{tabular}

FR - Fresenius crystalloid cardioplegic solution, ST - St. Thomas crystalloid cardioplegic solution, BL - Calafiore cold blood cardioplegic solution.

- hospitalization time longer than the median value for all patients (12 days) - "HOSP_time",

- vasoactive-inotropic score value higher than the median value of all patients (4.7) - "VIS" (vasoactive-inotropic score $\mathrm{mcg} / \mathrm{kg} / \mathrm{min}=\mathrm{DPA}+\mathrm{DBX}+100 \times \mathrm{ISO}+100 \times \mathrm{ADR}$ (where: DPA = dopamine, DBX = dobutamine, $I S O=$ isoprenaline, $A D R=$ adrenaline, score modified according to a local catecholamine administration policy) [7, 8]),

- the total amount of complications (including: acute renal failure requiring hemodiafiltration, pneumonia, cardiac arrhythmia, bleeding requiring reoperation, neurological complications, metabolic acidosis, pulmonary hypertension crisis and mediastinitis) - "Compl_count".
The aforementioned models were developed using the following explanatory variables:

- type of cardioplegia - blood cardioplegia (BL) vs. crystalloid cardioplegia ( $C R=$ St. Thomas cardioplegia + Fresenius cardioplegia) = "Cardiopl_double"; or blood cardioplegia vs. St. Thomas cardioplegia (ST) vs. Fresenius cardioplegia (FR) = "Cardiopl_triple"; both options were used interchangeably,

- patient's sex - "Sex",

- patient's age (in days) - "Age",

- type of congenital cardiac disease "CHD" (following cardiac diseases were considered: "AS/AI" - aortic valve diseases - complex and simple; "ASDII" - atrial septal defect, "VSD" - ventricular septal defect, "AVSD” - all types 
of atrioventricular septal defect (unbalanced forms were excluded), "ToF" - tetralogy of Fallot, "AVVI" - isolated atrioventricular valves diseases, "TGA" - transposition of great arteries excluding single ventricle, "UVH" - all spectrum of single ventricle heart, including hypoplastic left heart syndrome (HLHS), "MIX" - other, less frequent cardiac malformations),

- patient's body surface area $\left(\mathrm{m}^{2}\right)$ - "BSA",

- presence of concomitant genetic diseases - "Genetic" mainly Down's syndrome,

- surgeon performing the operation - "Surgeon",

- redo surgery - "REDO",

- urgent surgery - "Urgency",

- total operation time (min) - "OP_time",

- cardiopulmonary bypass time (min) - "CBP_time",

- aortic cross-clamp time ( $\mathrm{min}$ ) - "Ao-X-clamp_time",

- use of deep hypothermic circulatory arrest - "DHCA".

Continuous variables: BSA, Age, OP_time, CBP_time and Ao-X-clamp_time were discretized to quartiles in the majority of models.

\section{Development of statistical models}

For each dependent outcome variable a nonlinear multivariable regression model was created (Poisson version for "Compl_count" variable, binominal logistic version for the rest of variables). All independent variables entered the preliminary model. A genetic algorithm was used to search for the best model [9] by application of the Akaike information criterion [10]. A genetic algorithm is one of the best alternatives to the strategy of fitting all possible models, which definitely makes the computational time prohibitive even for recent computers. Conversely, a genetic algorithm explores only a subset of all possible models, randomly but with the preponderance towards the best model. This is achieved by simulation of biological processes: generation of the model's offspring with a mutation, recombination with another model and finally model selection. This makes genetic algorithms computationally fast. The leaveone-out cross-validation (LOOCV) method was used to validate the best model by its comparison to the preliminary model using a cross-validation estimate of prediction er-

Table IV. Model of vasoactive-inotropic score value. Model type: binomial

\begin{tabular}{|c|c|c|c|c|}
\hline Variable & $P$-value & Odds ratio & \multicolumn{2}{|c|}{$95 \% \mathrm{Cl}$} \\
\hline Intercept & 0.0033 & 0.2435 & 0.0932 & 0.6149 \\
\hline Cardioplegia: FR & 0.0416 & 1.5198 & 1.0165 & 2.2765 \\
\hline Cardioplegia: ST & 0.0140 & 1.6503 & 1.1078 & 2.4648 \\
\hline Age: 90-319 days & 0.0537 & 0.5785 & 0.3300 & 1.0058 \\
\hline Age: $319-860$ days & 0.6587 & 0.8406 & 0.3900 & 1.8262 \\
\hline Age: 860-999 days & 0.5105 & 1.4862 & 0.4652 & 4.9541 \\
\hline CHD type: ASDII & 0.4268 & 0.7333 & 0.3431 & 1.5910 \\
\hline CHD type: AVSD & 0.3386 & 1.4717 & 0.6686 & 3.2680 \\
\hline CHD type: AVVI & 0.0495 & 2.3700 & 1.0090 & 5.6761 \\
\hline CHD type: MIX & 0.1619 & 1.7387 & 0.8051 & 3.8086 \\
\hline CHD type: TGA & 0.8591 & 1.0910 & 0.4207 & 2.8950 \\
\hline CHD type: ToF & $<0.0001$ & 6.0350 & 2.6233 & 14.3336 \\
\hline CHD type: UVH & 0.2662 & 1.6706 & 0.6804 & 4.1770 \\
\hline CHD type: VSD & 0.6426 & 0.8423 & 0.4094 & 1.7523 \\
\hline BSA: $0.25-0.38 \mathrm{~m}^{2}$ & 0.8534 & 0.9514 & 0.5616 & 1.6192 \\
\hline BSA: $0.38-0.54 \mathrm{~m}^{2}$ & 0.0041 & 0.3245 & 0.1493 & 0.6951 \\
\hline BSA: $0.54-0.59 \mathrm{~m}^{2}$ & 0.0002 & 0.0986 & 0.0287 & 0.3228 \\
\hline Genetic & 0.1002 & 1.5265 & 0.9235 & 2.5360 \\
\hline Redo surgery & 0.0320 & 1.7736 & 1.0564 & 3.0173 \\
\hline CBP time: $54-86 \mathrm{~min}$ & $<0.0001$ & 4.6875 & 2.6491 & 8.5421 \\
\hline CBP time: $86-110 \mathrm{~min}$ & $<0.0001$ & 7.1750 & 3.9919 & 13.2737 \\
\hline CBP time: $110-126 \mathrm{~min}$ & $<0.0001$ & 13.0896 & 6.9434 & 25.4268 \\
\hline
\end{tabular}

Cross-validation estimate of prediction error: preliminary model: 0.179 , the best model: 0.157 .

CHD - type of congenital cardiac disease, CBP time - cardiopulmonary bypass time, FR - Fresenius crystalloid cardioplegic solution, ST - St. Thomas crystalloid cardioplegic solution, BSA - patient's body surface area, ASD II - atrial septal defect, AVSD - atrioventricular septal defect, AVVI - isolated atrioventricular valve malformations, MIX - other, less frequent cardiac malformations, TGA - all spectrum of transposition of great arteries, ToF - tetralogy of Fallot, UVH - all spectrum of single ventricle disease, including HLHS, VSD - ventricular septal defect, CI - confidence interval. 
ror. The smaller estimate of prediction error in the developed model than in the preliminary one signified the better performance of the former. The impact of an independent variable on an outcome variable (associated with the regression coefficient) was described by the odds ratio (OR) with $95 \%$ confidence interval $(\mathrm{Cl})$. Thus, the lower and upper confidence limits yielded an interval estimate of OR at $0.05 \alpha$. Predicting variables were considered significant at $p<0.05$.

\section{Statistical software}

The R Project (R: A Language and Environment for Statistical Computing, version 3.0.1) with the glmulti library implementing a genetic algorithm for the model search was used for statistical analysis [11]. Leave-one-out crossvalidation was performed using a boot library [12]. Because of time-consuming calculations, $\mathrm{R}$ batch scripts were created for automatic execution.

\section{Results}

The variable "Type of cardioplegia" entered into 3 out of 8 created models and with other explanatory variables had a statistically significant impact on the following de- pendent variables: vasoactive-inotropic score, low cardiac output syndrome presence, total amount of complications.

The model of vasoactive-inotropic score value in studied cohort was explained by the following predictors (Table IV):

- Increasing risk for vasoactive-inotropic score value higher than the cohort median:

- Type of cardioplegia - use of crystalloid Fresenius ( $p=$ $0.0416)$ and St. Thomas cardioplegia $(p=0.014)$,

- Atrioventricular valve defect $(p=0.0495)$, and tetralogy of Fallot presence $(p<0.001)$,

- Reoperation ( $p=0.032$ ),

- Cardiopulmonary bypass time longer than first quartile for studied population (CBP_time: in the second quartile: $p<0.001$; CBP_time: in the third quartile: $p<0.001$; CBP_time: in the fourth quartile: $p<0.001$ ),

- Decreasing risk for vasoactive-inotropic score value higher than the cohort median:

- Patient's body surface area greater than the median value (BSA in the third quartile: $p=0.0041$; BSA in the fourth quartile: $p=0.0002$ ),

The low cardiac output syndrome occurrence model was explained by the following predictors (Table V):

Table V. Model of low cardiac output syndrome occurrence. Model type: binomial

\begin{tabular}{|c|c|c|c|c|}
\hline Variable & $P$-value & Odds ratio & \multicolumn{2}{|c|}{$95 \% \mathrm{Cl}$} \\
\hline Intercept & $<0.0001$ & 0.0015 & 0.0001 & 0.0118 \\
\hline Cardioplegia: FR & 0.0015 & 3.3730 & 1.6436 & 7.4629 \\
\hline Cardioplegia: ST & $<0.0001$ & 5.0853 & 2.4728 & 11.3444 \\
\hline Age: 90-319 days & 0.1729 & 0.6369 & 0.3333 & 1.2250 \\
\hline Age: $319-860$ days & 0.2640 & 0.6440 & 0.2958 & 1.3932 \\
\hline Age: 860-999 days & 0.0017 & 0.2023 & 0.0720 & 0.5339 \\
\hline CHD type: ASDII & 0.1209 & 4.0899 & 0.7822 & 31.8598 \\
\hline CHD type: AVSD & 0.1684 & 3.1425 & 0.7327 & 22.1247 \\
\hline CHD type: AVVI & 0.0421 & 6.1369 & 1.2219 & 46.9253 \\
\hline CHD type: MIX & 0.0336 & 5.6853 & 1.3674 & 39.2556 \\
\hline CHD type: TGA & 0.1144 & 0.2409 & 0.0462 & 1.8487 \\
\hline CHD type: ToF & 0.1165 & 3.7052 & 0.8569 & 26.1832 \\
\hline CHD type: UVH & 0.0532 & 5.2252 & 1.1440 & 37.9075 \\
\hline CHD type: VSD & 0.1506 & 3.3172 & 0.7696 & 23.4585 \\
\hline Redo surgery & 0.0172 & 2.3611 & 1.1520 & 4.7631 \\
\hline Urgency & 0.0242 & 2.2657 & 1.1079 & 4.6131 \\
\hline Operation time: $150-205 \mathrm{~min}$ & 0.5381 & 0.7135 & 0.2461 & 2.1706 \\
\hline Operation time: $205-265 \mathrm{~min}$ & 0.0831 & 2.4927 & 0.9216 & 7.4218 \\
\hline Operation time: $265-960 \mathrm{~min}$ & 0.1600 & 2.2686 & 0.7442 & 7.4485 \\
\hline CBP time: $54-86$ min & 0.0360 & 4.3529 & 1.2307 & 20.8097 \\
\hline CBP time: 86-110 min & 0.0348 & 4.6372 & 1.2494 & 23.1424 \\
\hline CBP time: $110-126 \mathrm{~min}$ & 0.0004 & 14.2120 & 3.6971 & 73.0373 \\
\hline
\end{tabular}

Cross-validation estimate of prediction error: preliminary model: 0.09 , the best model: 0.087 .

CHD - type of congenital cardiac disease, CBP time - cardiopulmonary bypass time, FR - Fresenius crystalloid cardioplegic solution, ST - St. Thomas crystalloid cardioplegic solution, ASD II - atrial septal defect, AVSD - atrioventricular septal defect, AVVI - isolated atrioventricular valve malformations, MIX - other, less frequent cardiac malformations, TGA - all spectrum of transposition of great arteries, ToF - tetralogy of Fallot, UVH - all spectrum of single ventricle disease, including HLHS, VSD - ventricular septal defect, $\mathrm{CI}$ - confidence interval. 
- Increasing risk for LCOS in studied population:

- Type of cardioplegia - use of crystalloid Fresenius ( $p=$ $0.0015)$ and St. Thomas cardioplegia $(p<0.001)$,

- Presence of atrioventricular valve defect $(p=0.0421)$ and other less frequent cardiac malformations ( $p=$ 0.0336),

- Reoperation $(p=0.0172)$,

- Urgent operation ( $p=0.0242)$,

- Cardiopulmonary bypass time longer than first quartile of the cohort (CBP_time: second quartile: $p=$ 0.036; CBP_time: third quartile: $p=0.0348$; CBP_time in the fourth quartile: $p<0.001$ ),

- Decreasing risk for LCOS appearance in studied population:

- Patient's age in $4^{\text {th }}$ quartile of the cohort $(p=0.0017)$.

The model of the total amount of complications was explained by the following predictors (Table VI):

- Increasing the amount of complications:

- Type of cardioplegia - use of crystalloid St. Thomas cardioplegia ( $p=0.0002)$,

- Complex cardiac malformations presence (AVSD: $p=0.0099 ;$ AVVI: $p<0.001$ MIX: $p=0.0034$; ToF: $p<0.001 ; \mathrm{UVH}: p=0.003)$,

- Reoperation $(p<0.001)$,

- Urgent operation $(p=0.0126)$,
- Operation time, cardiopulmonary bypass time and patient's age - odds ratios not exceeding more than 1.0011 (OR and $\mathrm{Cl}-$ Table V),

- Decreasing the total amount of complications:

- Patient's body surface $(p<0.001)$.

The variable "Type of cardioplegia" entered into another two models explaining 30-day mortality and hospitalization time. However, the impact of used cardioplegia type was not statistically significant for these models (Tables VII and VIII).

Models of delayed sternal closure, mechanical ventilation time and intensive care unit stay time were created. However, the variable "Type of cardioplegia" did not enter into the mentioned models.

\section{Discussion}

This study shows that the type of cardioplegic solution, as well as the method of its administration, has a significant co-influence on selected postoperative clinical parameters such as vasoactive-inotropic score, low cardiac output syndrome presence and total amount of complications. These variables reflect postcardiotomy heart failure in children after CHD correction in a complementary way. The impact of cardioplegia type on these parameters described as the odds ratio ranges between 1.44 and 5.09 and is never dominant in relation to other explanatory variables in spe-

Table VI. Model of total amount of complications. Model: Poisson

\begin{tabular}{|c|c|c|c|c|}
\hline Variable & $P$-value & Odds ratio & & \\
\hline Intercept & 0.0902 & 0.4630 & 0.1791 & 1.0803 \\
\hline Cardioplegia: FR & 0.0555 & 1.2647 & 0.9984 & 1.6154 \\
\hline Cardioplegia: ST & 0.0002 & 1.5507 & 1.2338 & 1.9680 \\
\hline CHD type: ASDII & 0.9947 & 1.0027 & 0.4765 & 2.3668 \\
\hline CHD type: AVSD & 0.0099 & 2.6279 & 1.3431 & 5.9410 \\
\hline CHD type: AVVI & 0.0005 & 3.8997 & 1.9005 & 9.0694 \\
\hline CHD type: MIX & 0.0034 & 2.9466 & 1.5253 & 6.6075 \\
\hline CHD type: TGA & 0.9714 & 1.0139 & 0.5058 & 2.3294 \\
\hline CHD type: ToF & 0.0004 & 3.7040 & 1.9026 & 8.3398 \\
\hline CHD type: UVH & 0.0030 & 3.0527 & 1.5540 & 6.9189 \\
\hline CHD type: VSD & 0.0611 & 2.0139 & 1.0309 & 4.5478 \\
\hline Redo surgery & $<0.0001$ & 1.6633 & 1.3156 & 2.0846 \\
\hline Urgency & 0.0126 & 1.3473 & 1.0652 & 1.7014 \\
\hline Age & $<0.0001$ & 1.0011 & 1.0008 & 1.0014 \\
\hline BSA & $<0.0001$ & 0.0011 & 0.0003 & 0.0043 \\
\hline Operation time & $<0.0001$ & 1.0043 & 1.0034 & 1.0051 \\
\hline CBP time & 0.0324 & 1.0002 & 1.0000 & 1.0003 \\
\hline
\end{tabular}

Cross-validation estimate of prediction errors: preliminary model: 2.01, the best model: 1.96 .

CHD - type of congenital cardiac disease, CBP time - cardiopulmonary bypass time, FR - Fresenius crystalloid cardioplegic solution, ST - St. Thomas crystalloid cardioplegic solution, BSA - patient's body surface area, ASD II - atrial septal defect, AVSD - atrioventricular septal defect, AVVI - isolated atrioventricular valve malformations, MIX - other, less frequent cardiac malformations, TGA - all spectrum of transposition of great arteries, ToF - tetralogy of Fallot, UVH - all spectrum of single ventricle disease, including HLHS, VSD - ventricular septal defect, $\mathrm{Cl}$ - confidence interval. 
Table VII. Model of 30-day mortality. Model: binomial

\begin{tabular}{lcccc} 
Variable & P-value & Odds ratio & $95 \% \mathrm{Cl}$ & 0.0432 \\
\hline Intercept & $<0.0001$ & 0.0114 & 0.0023 & 2.5917 \\
\hline Cardioplegia: CR & 0.1920 & 1.4502 & 0.8443 & 2.2077 \\
\hline CHD type: ASDII & 0.3584 & 0.5035 & 0.1097 & 6.2581 \\
\hline CHD type: AVSD & 0.2404 & 1.9116 & 0.6924 & 5.3051 \\
\hline CHD type: AVVI & 0.7547 & 1.2537 & 0.2934 & 4.7490 \\
\hline CHD type: MIX & 0.5689 & 1.3914 & 0.4716 & 0.5864 \\
\hline CHD type: TGA & 0.0039 & 0.1542 & 0.0446 & 4.2233 \\
\hline CHD type: ToF & 0.7357 & 1.2201 & 0.4026 & 14.7541 \\
\hline CHD type: UVH & 0.0231 & 4.0588 & 1.2741 & 3.3931 \\
\hline CHD type: VSD & 0.9720 & 0.9795 & 0.3233 & 9.8320 \\
\hline Urgency & $<0.0001$ & 3.4288 & 3.0082 & 8.1218 \\
\hline CBP time: $54-86$ min & 0.2592 & 2.0555 & 0.6322 & 1.0011 \\
\hline CBP time: $86-110$ min & 0.0681 & 3.1152 & 4.5520 & 50.8774 \\
\hline CBP time: $110-126$ min & $<0.0001$ & 13.5062 & 0.5405 & 2.4160 \\
\hline DHCA & 0.7079 & 1.1532 & 0565 \\
\hline
\end{tabular}

Cross-validation estimate of prediction errors: preliminary model: 0.093 , the best model: 0.082 .

CHD - type of congenital cardiac disease, CBP time - cardiopulmonary bypass time, DHCA - use of deep hypothermic circulatory arrest, CR - crystalloid cardioplegia, ASD II - atrial septal defect, AVSD - atrioventricular septal defect, AVVI - isolated atrioventricular valve malformations, MIX - other, less frequent cardiac malformations, TGA - all spectrum of transposition of great arteries, ToF - tetralogy of Fallot, UVH - all spectrum of single ventricle disease, including HLHS, VSD - ventricular septal defect, $\mathrm{Cl}$ - confidence interval.

Table VIII. Model of hospitalization time. Model: binomial continuous

\begin{tabular}{|c|c|c|c|c|}
\hline Variable & $P$-value & Odds ratio & \multicolumn{2}{|c|}{$95 \% \mathrm{Cl}$} \\
\hline Intercept & 0.7933 & 0.9292 & 0.5364 & 1.6130 \\
\hline Cardioplegia: CR & 0.1194 & 0.7892 & 0.5856 & 1.0633 \\
\hline CHD type: ASDII & 0.0001 & 0.3252 & 0.1879 & 0.5594 \\
\hline CHD type: AVSD & 0.0011 & 0.3468 & 0.1825 & 0.6503 \\
\hline CHD type: AVVI & 0.7515 & 0.8865 & 0.4206 & 1.8787 \\
\hline CHD type: MIX & 0.0262 & 0.4906 & 0.2604 & 0.9160 \\
\hline CHD type: TGA & 0.2248 & 0.6002 & 0.2621 & 1.3662 \\
\hline CHD type: ToF & 0.8247 & 1.0695 & 0.5891 & 1.9398 \\
\hline CHD type: UVH & 0.1486 & 0.5763 & 0.2708 & 1.2131 \\
\hline CHD type: VSD & 0.1346 & 0.6728 & 0.3989 & 1.1289 \\
\hline Genetic & 0.0004 & 2.1924 & 1.4221 & 3.4082 \\
\hline Urgency & 0.0595 & 1.5593 & 0.9849 & 2.4866 \\
\hline Ao_X_clamp time & 0.0006 & 1.0099 & 1.0043 & 1.0157 \\
\hline DHCA & 0.1262 & 0.9773 & 0.9473 & 1.0052 \\
\hline
\end{tabular}

Cross-validation estimate of prediction errors: preliminary model: 0.272 , the best model: 0.229 .

CHD - type of congenital cardiac disease, Ao_X_clamp time - aortic cross-clamp time, DHCA - use of deep hypothermic circulatory arrest, CR - crystalloid cardioplegia, ASD II - atrial septal defect, AVSD - atrioventricular septal defect, AVVI - isolated atrioventricular valve malformations, MIX - other, less frequent cardiac malformations, TGA - all spectrum of transposition of great arteries, ToF - tetralogy of Fallot, UVH - all spectrum of single ventricle disease, including HLHS, VSD - ventricular septal defect, $\mathrm{Cl}$ - confidence interval.

cific models. This indicates the presence of more influential factors playing roles in aforementioned models as seen in a clinical context. However, cardioplegia type together with negative composition of other clinical variables (prolonged cardiopulmonary bypass time, low patient's body surface area, urgency of operation and complex CHD) can have a significant impact on postoperative course. In contrast to blood cardioplegia, crystalloid cardioplegic solutions have 
a significantly negative influence on postoperative course in presented regression models. This may suggest a similar, if not better, clinical result of cold blood cardioplegia administration. This observation corresponds with other reports $[13,14]$. Similarly, the influence of cardioplegia type was not dominant relatively to other clinical variables examined in this study.

Contrary to our findings, Young et al. [15] described less frequent inotropic drug administration in patients who received crystalloid cardioplegia. Similarly, Sinha et al. [16] reported shorter mechanical ventilation time and shorter hospital stay time in patients receiving crystalloid cardioplegia.

The cardioplegia type variable entered into the model of 30-day mortality, but without statistical significance. This observation is similar to other studies where type of used cardioplegia did not impact in-hospital mortality $[13,15]$.

Cardioplegia type was not a predictor in mechanical ventilation time and intensive care unit stay time models. It can be explained by a stronger influence of other noncardiac variables. They may affect the long ICU stay time due to prolonged mechanical ventilation (e.g. caused by pulmonary hypertension or pneumonia). It corresponds with other observations $[17,18]$ where type of cardioprotection has no influence on respiratory time, ICU stay time or hospitalization time. Similarly, the presence of delayed sternal closure (DSC) was not related to the type of used cardioplegia. It is comparable to other reports where the following variables may have a prevalent influence on DSC usage: cardiopulmonary bypass time, aortic cross clamp time, postoperative massive bleeding, severe preoperative patient's condition [19-21].

This study has several limitations. It is a retrospective observational study concerning a 6-year period. Intraoperative and postoperative procedures, quality of ICU care and surgeons' technique have evolved during that time. It could have affected the results of statistical analyses. The analysis of surgeon's influence on the studied variables showing a lack of impact may mitigate the bias connected with the operator's learning curve and the era of operation.

A certain drawback of the study may be connected with the multiplicity - creation of different models of clinical outcome based on the same set of independent variables. However, from the data-mining perspective this study tried to verify whether there is any influence of the most interesting variable - cardioplegia type - on our dependent variables that were not completely isolated one from another and were different manifestations of potentially inadequate cardioprotection.

The next limitation is the absence of a myocardial injury biomarker, whose postoperative blood levels could be correlated with type of used cardioplegia. These markers were not examined due to the retrospective character of the study. Moreover, myocardial injury biomarker release after operations with cardiopulmonary bypass and heart arrest makes their routine use difficult and confined to selected cases only [22, 23].

Better postoperative myocardial contractility was reported after blood cardioplegia administration [24, 25]. Due to the incompleteness of echocardiographic data this parameter was not included in the study.

\section{Conclusions}

Cardioprotection together with other factors influences postoperative clinical results in children with CHD. Contrary to crystalloid cardioplegia, blood cardioplegia does not adversely affect the outcome. Moreover, its application may have a protective effect by decreasing low cardiac output incidence and related complications.

\section{Disclosure}

Authors report no conflict of interest.

\section{References}

1. Turkoz R. Myocardial protection in pediatric cardiac surgery. Artif Organs 2013; 37: 16-20.

2. Robinson LA, Schwarz GD, Goddard DB, Fleming WH, Galbraith TA. Myocardial protection for acquired heart disease surgery: results of a national survey. Ann Thorac Surg 1995; 59: 361-372.

3. Bilfinger TV, Moeller JT, Kurusz M, Grimson RC, Anagnostopoulos CE. Pediatric myocardial protection in the United States: a survey of current clinical practice. Thorac Cardiovasc Surg 1992; 40: 214-218.

4. Kotani Y, Tweddell J, Gruber P, Pizarro C, Austin EH $3^{\text {rd }}$, Woods RK, Gruenwald C, Caldarone CA. Current cardioplegia practice in pediatric cardiac surgery: a North American multiinstitutional survey. Ann Thorac Surg 2013; 96 : 923-929.

5. Liu J, Feng Z, Zhao J, Li B, Long C. The myocardial protection of HTK cardioplegic solution on the long-term ischemic period in pediatric heart surgery. ASAIO J 2008; 54: 470-473.

6. Amark K, Berggren H, Bjork K, Ekroth A, Ekroth R, Nilsson K, Sunnegårdh J. Blood cardioplegia provides superior protection in infant cardiac surgery. Ann Thorac Surg 2005; 80: 989-994.

7. Gaies MG, Gurney JG, Yen AH, Napoli ML, Gajarski RJ, Ohye RG, Charpie JR, Hirsch JC. Vasoactive-inotropic score as a predictor of morbidity and mortality in infants after cardiopulmonary bypass. Pediatr Crit Care Med 2010; 11: 234-238.

8. Gaies MG, Jeffries HE, Niebler RA, Pasquali SK, Donohue JE, Yu S, Yu S, Gall C, Rice TB, Thiagarajan RR. Vasoactive-inotropic score is associated with outcome after infant cardiac surgery: an analysis from the Pediatric Cardiac Critical Care Consortium and Virtual PICU System Registries. Pediatr Crit Care Med 2014; 15: 529-537.

9. Xianyang W. An improved genetic algorithm adopting immigration operator. Intell Data Anal 2004; 8: 385-401

10. Wei P. Akaike's information criterion in generalized estimating equations. Biometrics 2001; 57: 120-125.

11. Calcagno V, de Mazanco C. glmulti: an R Package for easy automated model selection with (generalized) linear models. J Stat Softw 2010; 34: 1-29.

12. Canty A, Ripley B. boot: Bootstrap R (S-Plus) Functions. R package version 1.3-9. 2013 Available at: https://cran.r-project.org/web/packages/boot/citation.html

13. Guru V, Omura J, Alghamdi AA, Weisel R, Fremes SE. Is blood superior to crystalloid cardioplegia? A meta-analysis of randomized clinical trials. Circulation 2006; 114 (1 Suppl): I331-1338.

14. Ibrahim MF, Venn GE, Young CP, Chambers DJ. A clinical comparative study between crystalloid and blood-based St Thomas' hospital cardioplegic solution. Eur J Cardiothorac Surg 1999; 15: 75-83.

15. Young JN, Choy IO, Silva NK, Obayashi DY, Barkan HE. Antegrade cold blood cardioplegia is not demonstrably advantageous over cold crystalloid cardioplegia in surgery for congenital heart disease. J Thorac Cardiovasc Surg 1997; 114: 1002-1008.

16. Sinha P, Zurakowski D, Jonas RA. Comparison of two cardioplegia solutions using thermodilution cardiac output in neonates and infants. Ann Thorac Surg 2008; 86: 1613-1619.

17. Caputo M, Modi P, Imura H, Pawade A, Parry AJ, Suleiman MS, Angelini GD. Cold blood versus cold crystalloid cardioplegia for repair of ventricular septal 
defects in pediatric heart surgery: a randomized controlled trial. Ann Thorac Surg 2002; 74: 530-534.

18. Fang Y, Long C, Lou S, Guan Y, Fu Z. Blood versus crystalloid cardioplegia for pediatric cardiac surgery: a meta-analysis. Perfusion 2015; 30: 529-536.

19. Riphagen S, McDougall M, Tibby SM, Alphonso N, Anderson D, Austin C, Durward A, Murdoch IA. "Early" delayed sternal closure following pediatric cardiac surgery. Ann Thorac Surg 2005; 80: 678-684.

20. Erek E, Yalcinbas YK, Turkekul Y, Saygili A, Ulukol A, Sarioglu A, Sarioglu CT. Indications and risks of delayed sternal closure after open heart surgery in neonates and early infants. World J Pediatr Congenit Heart Surg 2012; 3: 229-235.

21. Tabbutt S, Duncan BW, McLaughlin D, Wessel DL, Jonas RA, Laussen PC. Delayed sternal closure after cardiac operations in a pediatric population. J Thorac Cardiovasc Surg 1997; 113: 886-893.
22. Zhang D, Jin S, Wei S, Wang X, Sun B. Troponin T during cardiopulmonary bypass. Zhonghua Wai Ke Za Zhi 2000; 38: 372-374.

23. Siaplaouras J, Thul J, Will JC, Bauer J, Kreuder J, Valeske K, et al. Cardiac troponin I after heart surgery corrective operation in infancy and childhood. Z Kardiol 2001; 90: 408-413.

24. Munch F, Purbojo A, Kellermann S, Janssen C, Cesnjevar RA, Ruffer A. Improved contractility with tepid modified full blood cardioplegia compared with cold crystalloid cardioplegia in a piglet model. Eur J Cardiothorac Surg 2015; 48: 236-243.

25. Iverson LI, Young JN, Ennix CL Jr, Ecker RR, Moretti RL, Lee J, et al. Myocardial protection: a comparison of cold blood and cold crystalloid cardioplegia. J Thorac Cardiovasc Surg 1984; 87: 509-516. 